\title{
FRACTIONAL HOLD CIRCUITS VERSUS POSITIVE REALNESS OF DISCRETE TRANSFER FUNCTIONS
}

\author{
M. DE LA SEN AND A. BILBAO-GUILLERNA
}

Received 25 October 2004

The appropriate use of fractional-order holds $(\beta$-FROH) of correcting gains $\beta \in[-1,1]$ as an alternative to the classical zero-and first-order holds ( $\mathrm{ZOHs}, \mathrm{FOHs}$ ) is discussed related to the positive realness of the associate discrete transfer functions obtained from a given continuous transfer function. It is proved that the minimum direct input/output gain (i.e., the quotient of the leading coefficients of the numerator and denominator of the transfer function) needed for discrete positive realness may be reduced by the choice of $\beta$ compared to that required for discretization via $\mathrm{ZOH}$.

\section{1. $\beta$-fractional-order holds and introductory background on positive realness}

The realizable continuous transfer function $p(s)=q(s) / n(s)=p^{\prime}(s)+d$, of numerator and denominator polynomials $q(s)$ and $n(s)$ with $p^{\prime}(s)$ being strictly proper, is positive real $(p \in\{P R\})$ if $p(s) \in \mathbb{R}$ (the set of real numbers), for all $s \in \mathbb{R}$ and $\operatorname{Re}(p(s)) \geq 0$ for $\sigma=\operatorname{Re} s \geq 0$, for all $s \in \mathbb{C}[3,4]$ (the set of complex numbers). A necessary condition for a realizable continuous transfer function to be positive real is that it is stable with zero or unity relative degree and with eventually critically stable poles being simple with nonnegative residuals. Positive realness also implies stability of zeros $[1,2,4]$ and it is a key feature in achieving asymptotic hyperstability via feedback for all nonlinear/time-varying device satisfiying a Popov -type inequality [5]. The scalar $d$ is the direct input/output gain, with $d=0$ if and only if $p(s)=p^{\prime}(s)$ is strictly proper. Consider the class of $\beta$-FROH (including $\mathrm{ZOH}(\beta=0)$ and $\mathrm{FOH}(\beta=1))$ of transfer function $h_{\beta}(s)$ leading to the $\beta$-dependent discrete transfer functions

$$
\begin{aligned}
& g_{\beta}(z)=Z\left[h_{\beta}(s) p(s)\right]=g_{\beta}^{\prime}(z)+d_{\beta}, \\
& h_{\beta}(s)=\left[1-\beta+\beta \frac{1+s T}{T} h_{0}(s)\right] h_{0}(s),
\end{aligned}
$$

where $Z[\cdot]$ stands for the $z$-transform. The transfer function $h_{\beta}(s)$ is obtained directly $[1,2]$ since the output of the hold device being injected as input to the continuous transfer 
function is

$$
u(t)=u_{k}+\frac{\beta}{T}\left(u_{k}-u_{k-1}\right)(t-k T)
$$

for all $t \in[k T,(k+1) T)$ with $u_{k}=u(k T)$ for any sample-indicator integer $k \geq 0$ with $T$ being the sampling period. Note that $h_{\beta}(s)$ may be directly synthesized with two ZOHs and a simple linear network. It has been proved [4] that $g_{0}(z)$ is discrete positive real $\left(g_{0} \in\right.$ $\left\{P R_{d}\right\}$ ) if $p(s)$ is stable (or, in particular, positive real) and biproper (i.e., of zero relative degree) with a sufficiently large associated direct input/output gain $d_{0}=d \geq d_{\min }>0$. This implies that if $d=0$ (i.e., $p(s)=p^{\prime}(s)$ is strictly proper), then $g_{0} \notin\left\{P R_{d}\right\}$ even if $p \in\{P R\}$ with unity relative degree. Positive realness under discretization via $\beta$-FROH is now discussed by first defining positive realness with prescribed margins.

Definition 1.1. It is said that $g_{\beta} \in\left\{P R_{d}(\varepsilon)\right\}$, some $\varepsilon \geq 0$, if $\operatorname{Re} g_{\beta}(z) \geq \varepsilon$ for all $z \in U C:=$ $\{z \in \mathbb{C}:|z|=1\}$.

Note that $\left\{P R_{d}(0)\right\} \equiv\left\{P R_{d}\right\}, g_{\beta} \in\left\{P R_{d}(\varepsilon)\right\} \Rightarrow g_{\beta} \in\left\{P R_{d}\left(\varepsilon^{\prime}\right)\right\}$ for all $\varepsilon^{\prime} \in[0, \varepsilon)$ and $g_{\beta} \in\left\{P R_{d}(\varepsilon)\right\}$ for some real $\varepsilon>0 \Rightarrow g_{\beta} \in\left\{S P R_{d}\right\}$, that is, $g_{\beta}(z)$ is strictly positive real (since $\left.\operatorname{Min}_{z \in U C}\left(\operatorname{Re} g_{\beta}(z)\right)>0\right)$.

\section{Positive realness of $g_{\beta}(z)$}

Direct simple calculations allow rewriting the first equation in (1.1) as

$$
g_{\beta}(z)=\left(1-\beta z^{-1}\right)\left(g_{0}^{\prime}(z)+d_{\beta}\right)+\beta T^{-1} z^{-1}(z-1) g_{01}(z)
$$

if $p(s)=p^{\prime}(s)+d_{\beta}$ with $g_{01}(z)=\left(1-z^{-1}\right) Z\left(s^{-2} p(s)\right)$ which implies that $g_{0}(z)=(1-$ $\left.\beta z^{-1}\right)\left(g_{0}^{\prime}(z)+d_{0}\right)$. Simple calculations with (2.1) lead to

$$
\begin{aligned}
g_{\beta}(z) & =(1+\beta \tilde{g}(z))\left(g_{0}^{\prime}(z)+d_{\beta}\right), \\
\tilde{g}(z) & =\frac{1}{z}\left[\frac{1}{T} \frac{q_{01}(z)}{q_{0}(z)}-1\right]
\end{aligned}
$$

since $g_{01}(z) / g_{0}(z)=q_{01}(z) /\left((z-1) q_{0}(z)\right)$ with $q_{01}(z)$ and $q_{0}(z)$ being the respective numerator polynomials of $g_{01}(z)$ and $g_{0}(z)$ since their respective denominator polynomials $n_{01}(z)$ and $n_{0}(z)$ satisfy the constraint $n_{01}(z)=(z-1) n_{0}(z)$ from direct calculations involving $z$-transforms. Since $p^{\prime}(s)$ is strictly proper, then $g_{\beta}^{\prime}(z)=Z\left[h_{\beta}(s) p^{\prime}(s)\right]$ is strictly proper of unity relative degree and order $\operatorname{deg}(n(s))$ if $\beta=0$ and $(1+\operatorname{deg}(n(s)))$ if $\beta \neq 0$. Let real constants $m_{i}, m_{s} \geq m_{i} ; \tilde{m}_{i}, \tilde{m}_{s} \geq \tilde{m}_{i}$ be such that

$$
\operatorname{Re} \tilde{g}(z) \in\left[\tilde{m}_{i}, \tilde{m}_{s}\right], \quad \operatorname{Re}\left(\tilde{g}(z) g_{0}^{\prime}(z)\right) \in\left[m_{i}, m_{s}\right], \quad \forall z \in U C .
$$

Direct calculations using the worst lower-bound minimum bound for $\operatorname{Re}\left(g_{\beta}(z)\right)$ from (2.2) via (2.3) lead to

$$
\begin{aligned}
& \operatorname{Reg}_{\beta(\geq 0)}(z) \geq \varepsilon_{0}+\Delta d_{\beta}+\beta\left[m_{i}+\left(d_{0}^{\prime}+\varepsilon_{0}+\Delta d_{\beta}\right) \tilde{m}_{i}\right] \\
& \operatorname{Re} g_{\beta(<0)}(z) \geq \varepsilon_{0}+\Delta d_{\beta}-|\beta|\left[m_{s}+\left(d_{0}^{\prime}+\varepsilon_{0}+\Delta d_{\beta}\right) \tilde{m}_{s}\right]
\end{aligned}
$$


which hold, respectively, for $\beta \geq 0$ and for $\beta<0$. The technical subsequent assumption is then used.

Assumption 2.1. $g_{0} \in\left\{P R_{d}(\varepsilon)\right\}\left(\Rightarrow g_{0} \in\left\{P R_{d}\right\}\right)$ and $d_{\beta} \geq\left(d_{o}-\varepsilon_{0}\right)$ for some real $\varepsilon_{0} \geq 0$, all $\beta$-FROH.

Now, define auxiliary real constants $\bar{m}_{\ell}$ from $m_{\ell}, \tilde{m}_{\ell}$ from $(2.3)$ as $\bar{m}_{\ell}:=m_{\ell}+\left(d_{0}^{\prime}+\right.$ $\left.\varepsilon_{0}\right) \tilde{m}_{\ell}(\ell=i, s)$. From Assumption 2.1 and the constraints (2.4), the following result holds.

Theorem 2.2 (discrete positive realness via design of $\beta$ ). If Assumption 2.1 holds, then $g_{\beta} \in\left\{P R_{d}(\varepsilon)\right\}$ with $\operatorname{Re}_{\beta}(z) \geq \varepsilon$ for some sufficiently small $\varepsilon \geq 0$ and some $\beta-F R O H, \beta \in$ $\left[\beta_{\min }, \beta_{\max }\right] \subseteq[-1,1]$ if some of the subsequent items hold.

(i)

$$
\left|\frac{\varepsilon-\varepsilon_{0}-\Delta d_{\beta}}{\bar{m}_{i}}\right| \leq \beta \leq 1
$$

provided that

$$
\varepsilon-\varepsilon_{0} \geq \Delta d_{\beta} \geq \operatorname{Max}\left(-\varepsilon_{0},-\frac{\bar{m}_{i}}{\tilde{m}_{i}}\right) \quad \text { if } \tilde{m}_{i} \neq 0,
$$

or

$$
\varepsilon-\varepsilon_{0} \geq \Delta d_{\beta} \geq-\varepsilon_{0} \quad \text { if } \bar{m}_{i}>0, \quad \tilde{m}_{i} \neq 0 .
$$

(ii)

$$
0 \leq \beta \leq \operatorname{Max}\left(\left|\frac{\varepsilon-\varepsilon_{0}-\Delta d_{\beta}}{\bar{m}_{i}}\right|, 1\right)
$$

provided that

$$
-\frac{\bar{m}_{i}}{\tilde{m}_{i}}>\Delta d_{\beta} \geq \varepsilon-\varepsilon_{0} \quad \text { if } \tilde{m}_{i} \neq 0,
$$

or

$$
\Delta d_{\beta} \geq \varepsilon-\varepsilon_{0} \quad \text { if } \bar{m}_{i}<0, \quad \tilde{m}_{i}=0 .
$$

(iii)

$$
\beta<0, \quad|\beta| \leq \operatorname{Max}\left(\left|\frac{\varepsilon+\Delta d_{\beta}-\varepsilon_{0}}{\bar{m}_{s}}\right|, 1\right)
$$

provided that

$$
\Delta d_{\beta} \geq \operatorname{Max}\left(\varepsilon-\varepsilon_{0},-\frac{\bar{m}_{s}}{\tilde{m}_{s}}\right) \quad \text { if } \tilde{m}_{s} \neq 0
$$

or

$$
\Delta d_{\beta} \geq \varepsilon-\varepsilon_{0} \quad \text { if } \bar{m}_{s}>0, \quad \tilde{m}_{s} \neq 0 .
$$


(iv)

$$
\beta<0, \quad 1 \geq|\beta| \geq\left|\frac{\varepsilon_{0}+\Delta d_{\beta}-\varepsilon}{\bar{m}_{s}}\right|
$$

provided that

$$
\varepsilon-\varepsilon_{0} \geq \Delta d_{\beta} \geq \operatorname{Max}\left(-\varepsilon_{0},-\frac{\bar{m}_{s}}{\tilde{m}_{s}}\right) \quad \text { if } \tilde{m}_{s} \neq 0,
$$

or

$$
\varepsilon-\varepsilon_{0} \geq \Delta d_{\beta} \geq-\varepsilon_{0} \quad \text { if } \bar{m}_{s}<0, \quad \tilde{m}_{s} \neq 0
$$

By using (2.4) with $\varepsilon=\varepsilon_{0}$, the following result stands.

Theorem 2.3 (positive realness via $\beta$-FROH by increasing/decreasing direct input/output gains). If $g_{0} \in\left\{P R_{d}\left(\varepsilon_{0}\right)\right\}$ with $d_{0}=d_{0}^{\prime}+\varepsilon_{0}$, then $g_{\beta} \in\left\{P R_{d}\left(\varepsilon_{0}\right)\right\}$ if $d_{\beta}=d_{0}+\Delta d_{\beta}$ with

$$
\begin{aligned}
& \Delta d_{\beta} \geq \operatorname{Max}\left(-\varepsilon_{0},-\frac{\beta \bar{m}_{i}}{1+\beta \widetilde{m}_{i}}\right) \quad \text { if } \beta \in[0,1] \text { with } \beta \neq-\frac{1}{\tilde{m}_{i}}, \\
& \Delta d_{\beta} \geq \operatorname{Max}\left(-\varepsilon_{0}, \frac{|\beta| \bar{m}_{s}}{1-|\beta| \tilde{m}_{s}}\right) \quad \text { if } \beta \in[-1,0] \text { with }|\beta| \neq \frac{1}{\tilde{m}_{s}} .
\end{aligned}
$$

Remark 2.4. Note that the margin of positive realness, compared to that achieved with a $\mathrm{ZOH}$, is improved with smaller positive values $0<d_{\beta}<d_{0}$, since for positive realness of discrete transfer functions, the relative degree is required to be zero, the direct input/ output gain from Theorem 2.3 if $\beta<0$ satisfies $|\beta|<\operatorname{Min}\left(1,1 /\left|\tilde{m}_{s}\right|\right)$ provided that $\operatorname{Min}\left(\varepsilon_{0}, \tilde{m}_{s}\right)>0$. This also holds if $1 \geq \beta>1 /\left|\tilde{m}_{i}\right|$ with $\tilde{m}_{i}<0,\left|\tilde{m}_{i}\right|>1$, and $\bar{m}_{i}<0$, or if $0<\beta \leq \operatorname{Min}\left(1 /\left|\tilde{m}_{i}\right|, 1\right)$ if $\left|\tilde{m}_{i}\right|<1$. If the usual constraint $\beta \in[-1,1]$ is removed, then several alternative solutions with $|\beta|>1$ are useful for such a purpose of achieving positive realness for $0<d_{\beta}<d_{0}$.

Example 2.5. Note that Theorems 2.2 and 2.3 are based on obtaining worst-case positive lower bounds of the $\operatorname{Re}\left(g_{\beta}(z)\right)$, where each $\beta$-dependent right-hand side term in $(2.4)$ is minimized. However, it is possible to obtain refinements from positive lower bounds via numerical evaluation of the relation

$$
d(\beta)>d_{\min }(\beta):=-\left|\operatorname{Min}_{z \in U C} g_{\beta}^{\prime}(z)\right|=-\left|\operatorname{Min}_{z \in U C} Z\left[h_{\beta}(s) p^{\prime}(s)\right]\right| \geq 0
$$




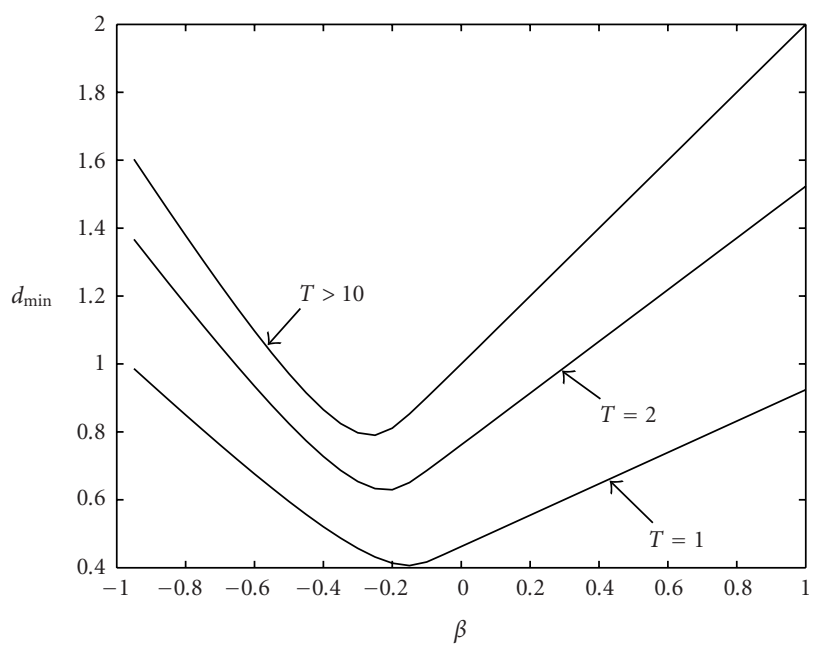

Figure 2.1

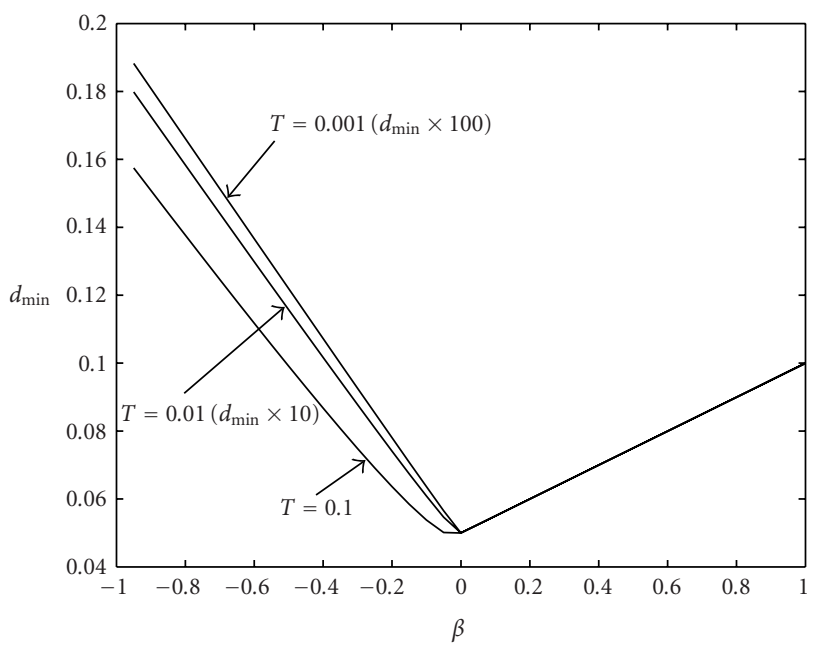

Figure 2.2

Proceed in that way with $p^{\prime}(s)=1 /(s+1) \in\{P R\}$. Figures 2.1 and 2.2 display the threshold $d_{\min }(\beta)$ to be used in the continuous transfer function to achieve positive realness with a $\beta$-FROH for six distinct values of the sampling period ranging from 0.001 to 10 seconds. Note that the smaller values of such a threshold are highly dependent on the sampling period and achieved for a range of negative values of $\beta$ which improve the threshold $d_{\min }(0)$ required for $\beta=0$. 


\section{References}

[1] S. Liang and M. Ishitobi, The stability properties of the zeros of sampled models for time delay systems in fractional order hold case, Dyn. Contin. Discrete Impuls. Syst. Ser. B Appl. Algorithms 11 (2004), no. 3, 299-312.

[2] S. Liang, M. Ishitobi, and Q. Zhu, Improvement of stability of zeros in discrete-time multivariable systems using fractional-order hold, Internat. J. Control 76 (2003), no. 17, 1699-1711.

[3] M. de la Sen, A method for general design of positive real functions, IEEE Trans. Circuits Systems I Fund. Theory Appl. 45 (1998), no. 7, 764-769.

[4]_, Relationships between positive realness of continuous transfer functions and their digital counterparts, Electron. Lett. 35 (1999), no. 16, 1298-1299.

[5] _ On the asymptotic hyperstability of dynamic systems with point delays, IEEE Trans. Circuits Systems I Fund. Theory Appl. 50 (2003), no. 11, 1486-1488.

M. de la Sen: Instituto de Investigación y Desarrollo de Procesos, Facultad de Ciencias, Universidad del País Vasco Leioa (Bizkaia), Apartado 644 de Bilbao, 48080 Bilbao, Spain

E-mail address: wepdam@lg.ehu.es

A. Bilbao-Guillerna: Instituto de Investigación y Desarrollo de Procesos, Facultad de Ciencias, Universidad del País Vasco Leioa (Bizkaia), Apartado 644 de Bilbao, 48080 Bilbao, Spain

E-mail address: webbigua@ehu.es 


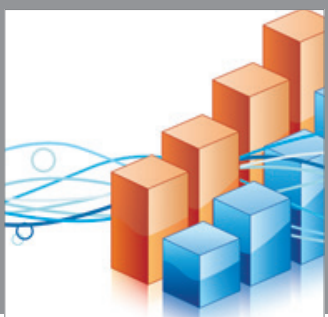

Advances in

Operations Research

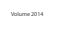

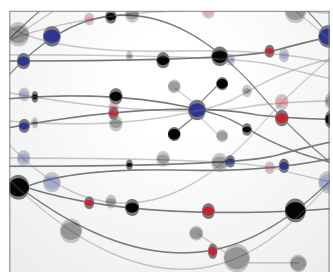

\section{The Scientific} World Journal
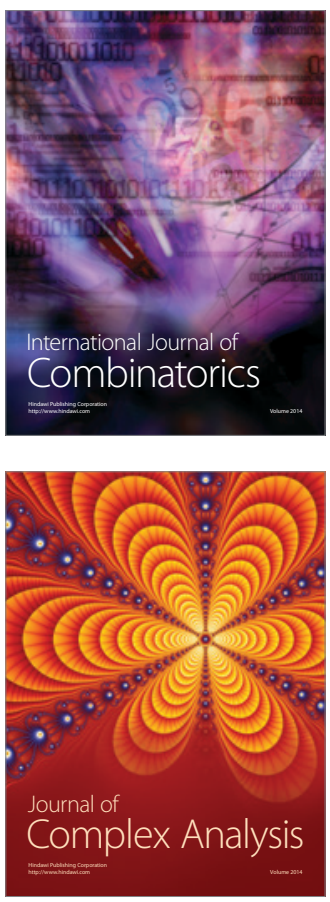

International Journal of

Mathematics and

Mathematical

Sciences
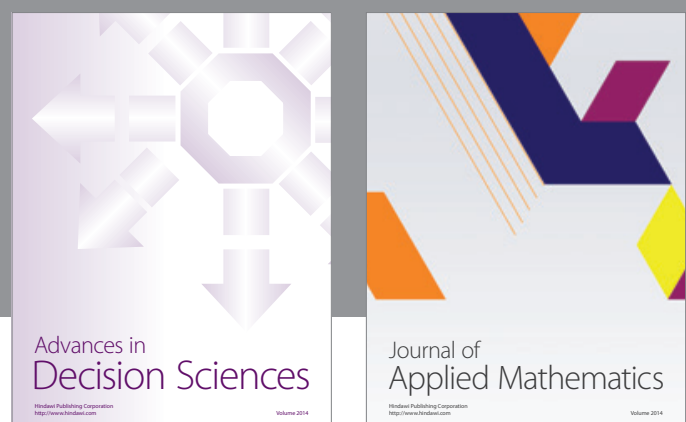

Journal of

Applied Mathematics
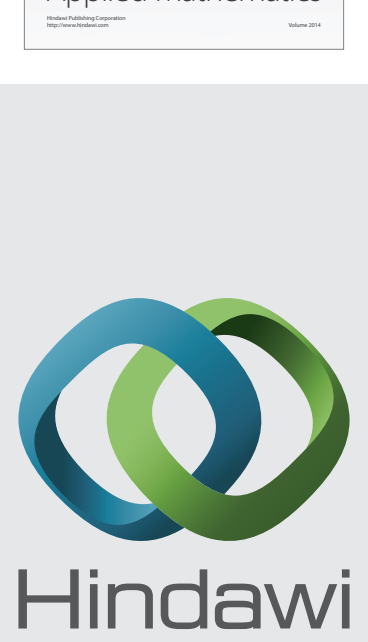

Submit your manuscripts at http://www.hindawi.com
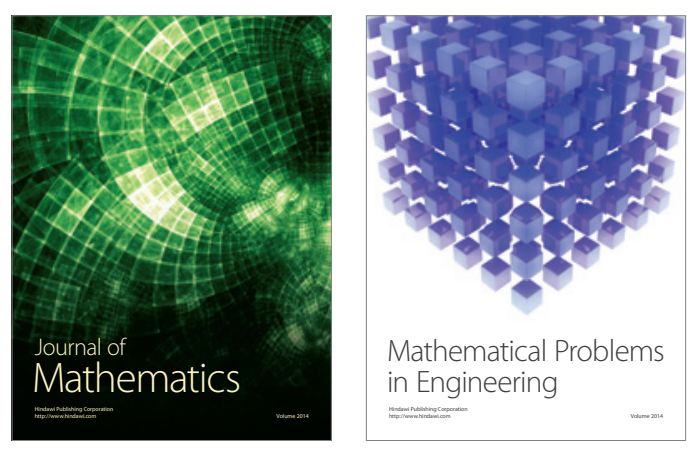

Mathematical Problems in Engineering
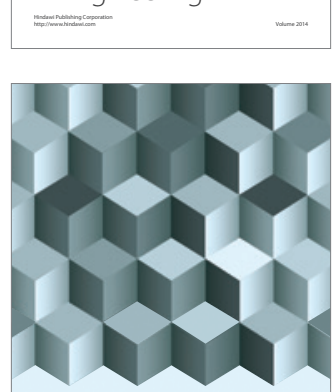

Journal of

Function Spaces
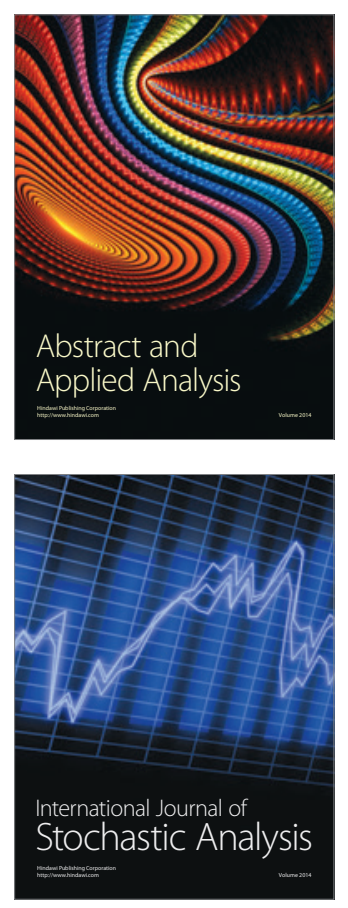

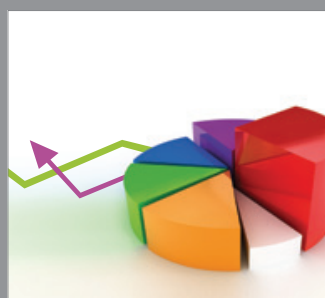

ournal of

Probability and Statistics

Promensencen
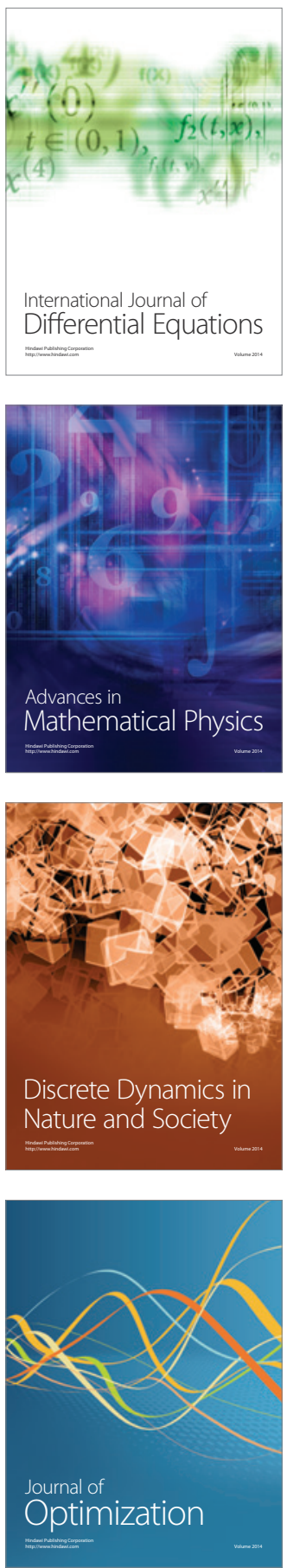